\title{
COMPARISON OF RESULTS OF TEXTURE MEASUREMENTS OBTAINED BY X-RAY AND BY NEUTRON DIFFRACTION
}

\author{
O. ENGLER, ${ }^{1}$ J. PALACIOS, ${ }^{2}$ W. SCHÄFER,$^{3}$ E. JANSEN,$^{3}$ K. LÜCKE ${ }^{1}$ \\ and G. WILL ${ }^{3}$
}

${ }^{I}$ Institut für Metallkunde und Metallphysik, RWTH Aachen, Kopernikusstr. 14, W-5100 Aachen, Germany

${ }^{2}$ Escuela Superior de Fisica y Matematicas, I.P.N. Apdo. Postal 75-544, 07300 México, D.F., México

${ }^{3}$ Mineralogisches Institut der Universität Bonn, Poppelsdorfer SchloB, W-5300 Bonn, Germany

(4 April, 1992)

\begin{abstract}
Texture measurements were carried out on $95 \%$ cold rolled and also on recrystallized high purity copper sheets by means of X-ray and neutron diffraction. The purpose of this study was to compare the results obtained by the two different measuring techniques and also to test the accuracy of the corrections normally used for deriving pole figures from X-ray data. In sheets containing texture inhomogeneities, X-ray pole figures were measured at different distances from the surface and two methods of producing averaged pole figures comparable to neutron measurements were applied. From the X-ray and from the neutron determined pole figures orientation distribution functions (ODF's) were calculated. The resulting differences which are less than $10 \%$ are discussed.
\end{abstract}

KEY WORDS X-ray diffraction, neutron diffraction, texture measuring techniques, copper.

\section{INTRODUCTION}

Quantitative texture determinations of polycrystalline metallic materials are mostly carried out by X-ray back reflection techniques. The resulting pole figure data, however, require corrections for errors due to background irradiation absorption, defocusing of X-rays, and due to effects of sample geometry. The background is generally determined from measurements outside, but near to the Bragg peaks, whereas the other three effects are corrected by measuring a textureless (e.g. powder) sample of equal shape. Up to now, no thorough quantitative test of the accuracy and reliability of such corrections has been performed. A good way for such a check is to compare results obtained by X-rays to those of neutron measurements applied to the same sample.

Contrary to X-rays, the transparency of matter for neutrons is very high. For most elements the absorption coefficient for neutrons is smaller by about four orders of magnitude than that for X-rays (Wenk et al., 1984, Bunge, 1989). Therefore, in neutron diffraction, pole figures can be measured in transmission. If spherical samples are used, no corrections with respect to beam geometry are necessary as shown by Tobisch and Bunge (1972). Even if the shape of the sample 
deviates slightly from a sphere, being a cube or a cylinder for example (which would simplify the sample preparation), these corrections are negligible. Since, furthermore, no defocusing effects have to be considered, only the background which for each individual pole figure can be determined very properly has to be subtracted.

The aim of the present investigation is a quantitative comparison of pole figures and orientation distribution functions (ODF's) obtained from X-ray and neutron diffraction data of the same copper sheet. This should also give information on the quality of the corrections applied in X-ray work. A true comparison of the measuring techniques-X-ray and neutron diffraction-was not possible due to the quite large number of factors being varied during the present study.

\section{EXPERIMENTAL PROCEDURE}

A high purity copper sheet with initially $35 \mu \mathrm{m}$ grain size was cold rolled with a thickness reduction of $95 \%$ to $0.9 \mathrm{~mm}$ thickness in a way that should lead to a homogeneous texture (Hammelrath et al., 1991).

From this sheet, plates of $14 \times 24 \mathrm{~mm}^{2}$ for $X$-ray and of $10 \times 10 \mathrm{~mm}^{2}$ for neutron diffraction were cut and etched in $\mathrm{HNO}_{3}$ to remove surface layers. Some of the plates were recrystallization annealed for 20 minutes at $300^{\circ} \mathrm{C}$.

$X$-ray diffraction texture measurements were performed in $(\alpha \times \beta=) 5^{\circ} \times 5^{\circ}$ steps at the automated high speed texture goniometer of the Institut für Metallkunde und Metallphysik of the RWTH Aachen (for details see Hirsch et al., 1986). The corrections of the X-ray data mentioned above were applied by using a fine grained textureless copper powder sample.

Subsequently, the pole figure data $I_{h k l}(\alpha, \beta)$ were pseudo-normalized by a factor $N_{i}$ with

$$
N_{i}=\frac{\sum_{\alpha_{\min }}^{\alpha_{\max }} \sum_{\beta=0}^{\beta=360^{\circ}} I_{h k l}(\alpha, \beta) \cdot\left(\cos \left(\alpha-\frac{\Delta \alpha}{2}\right)-\cos \left(\alpha+\frac{\Delta \alpha}{2}\right)\right) \cdot \Delta \beta}{2 \cdot \pi \cdot\left(\cos \left(\alpha_{\min }-\frac{\Delta \alpha}{2}\right)-\cos \left(\alpha_{\max }+\frac{\Delta \alpha}{2}\right)\right)}
$$

and plotted in the stereographic projection.

In order to take into account possible through thickness inhomogeneities of the samples, texture measurements of the as rolled samples were made at four different distances from the surface, namely for the depth parameters $s=0.0$, $0.3,0.6$ and 0.9 . (Here the parameter $s=0$ indicates the central and $s=1$ the surface layer of the sample.) Planes at these depths were obtained by stepwise removing the surface layers by grinding and etching. The recrystallized samples were found to be much more homogeneous through thickness, so that only the layers $s=0$ and $s=0.9$ were investigated.

Furthermore, in some samples a new surface was prepared at an angle to the original sheet surface. As is demonstrated in Figure 1, the thickness of the sheet was reduced at one edge of the sample from $0.9 \mathrm{~mm}$ to $0.45 \mathrm{~mm}$. For the sample $24 \mathrm{~mm}$ long this yields an angle of $\approx 1^{\circ}$. At such an angle definitely not being harmful to the resulting description of orientations the new surface contains all layers from $s=0$ to $s=1$. This means that under irradiation all layers are covered 


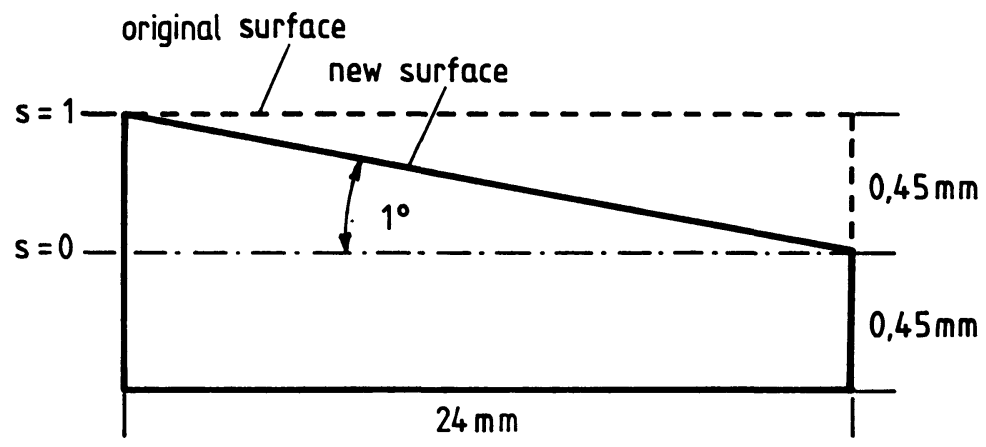

Figure 1 Scheme of preparing slanted surfaces for averaging inhomogeneous textures (not drawn to scale).

equally by the incident X-ray beam and thus a texture averaged over the various layers of the sheet is obtained. This should be directly comparable to the neutron results.

In order to measure textures by neutron diffraction, eleven of the $1 \mathrm{~cm}^{2}$ plates were glued on top of each other in the same orientation. Finally the corners and edges of the resulting cube with $1 \mathrm{~cm}$ edge length were rounded. For such samples neutron diffraction pole figures have been measured with the University of Bonn texture diffractometer at the reactor FRJ-2 in the Forschungszentrum Jülich (Schäfer et al., 1988). This diffractometer is equipped with the position sensitive detector (PSD) JULIOS (for details see Schäfer et al., 1986, and Will et al., 1989). Instead of measuring individual pole figures only in the peak maximum positions $2 \theta$ like in the $X$-ray experiments, the PSD records a large section of the diffraction diagram of about $25^{\circ}$ in $2 \theta$ containing simultaneously complete peak profiles of different $h k l$ 's. The pole figures are composed of about 800 different sample orientations between which the intensities are interpolated. A background function is adjusted to all individual data sets, so that subsequently for all sample orientations the actual background can be subtracted.

For both techniques of measurements, ODF's were calculated from four incomplete pole figures $(\{111\},\{200\},\{220\}$ and $\{113\})$ by means of the series expansion method with $l_{\max }=22$ (Bunge, 1969). For the neutron experiments, first a coordinate transformation is necessary, by which the angles $\omega, \chi$ and $\varphi$ of the Eulerian cradle are transformed into the pole figure angles $\alpha$ and $\beta$ (Bunge, Wenk and Pannetier, 1982). Points which are missing for the "equal-angular-scan" were linearly interpolated. Although by the PSD-technique complete pole figure data sets were delivered by additional measuring points covering "blind areas", only the data with $5^{\circ} \leq \alpha \leq 85^{\circ}$ corresponding to incomplete pole figures of the $\mathrm{X}$-ray experiments were used in order to obtain a better comparison. Also the same computer program was applied for both techniques.

\section{RESULTS}

\section{(a) Rolled Samples}

Figure 2 exhibits the microstructure of the rolled copper sheet, being obtained by an orientation sensitive etching technique ( $\{111\}$-etch, see Köhlhoff, Sun and 


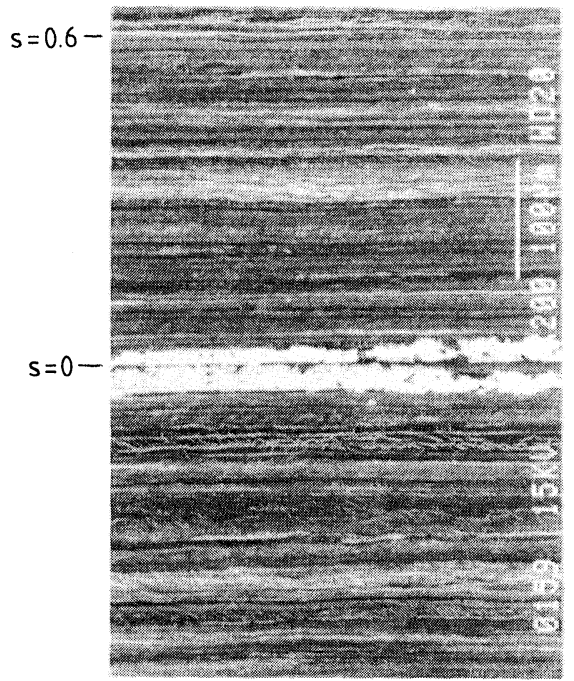

a)

$$
200 x
$$

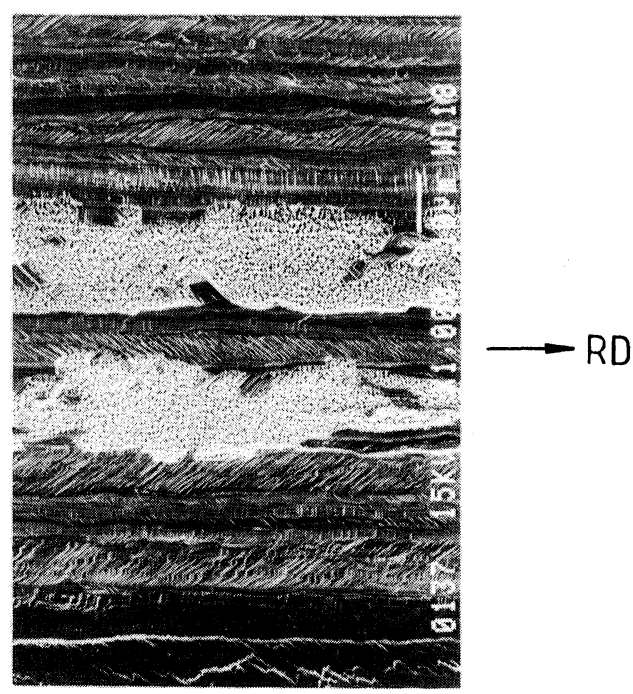

b)

Figure 2 Microstructure of the rolled copper sample (longitudinal sections): a) $200 \times$, b) $1000 \times$.

Lücke, 1987). The figure shows a typical deformation structure. Near the mid layer, however, some white regions can be observed (Figure 2a), which under higher magnification (Figure $2 b$ ) show the etching pattern of the cube orientation $\{001\}\langle 100\rangle$.

The $\{111\} \mathrm{X}$-ray diffraction pole figures of the rolled sheet are shown in Figure 3 for the layers $s=0,0.3,0.6$, and 0.9 . They are typical for rolled copper, but, surprisingly exhibit a certain inhomogeneity through the sheet thickness, despite of being homogeneously rolled. The largest difference stems from the region near $s=0$, where a strong cube component appears. In Figure 4 the corresponding ODF's are represented by sections $\varphi_{2}$-constant through the Euler angle space $\left(\varphi_{1}, \phi, \varphi_{2}\right)$. They, too, exhibit the typical copper type rolling texture with the orientations mainly assembled along an orientation fibre containing the Copper $\{112\}\langle 111\rangle, S\{123\}\langle 634\rangle$ and Brass $\{011\}\langle 211\rangle$ orientation (see Hirsch and Lücke, 1988). Here the different intensities of the cube $\left(0^{\circ}, 0^{\circ}, 0^{\circ}\right)$ orientation can very clearly be recognized. Figures $5 \mathrm{a}, 6 \mathrm{a}$ show the mathematically averaged texture obtained by simply adding and re-normalizing the pole figures of the four layers with

$$
I_{\mathrm{ave}}=\left(I_{s=0}+I_{s=0.3}+I_{s=0.6}+I_{s=0.9}\right) / 4
$$

Figures $5 b, 6 b$ give the experimentally averaged texture stemming from a sample with the slanted surface (see Figure 1). Only minor differences are found between these two types of averaging. Particularly the cube intensity is higher in Figures $5 a, 6 a$ derived from the four layers averaged.

In Figure $7 \mathrm{a}, \mathrm{b}$ the corresponding incomplete $\{111\}$ pole figure as well as the ODF obtained from the neutron measurements are presented. Since the 

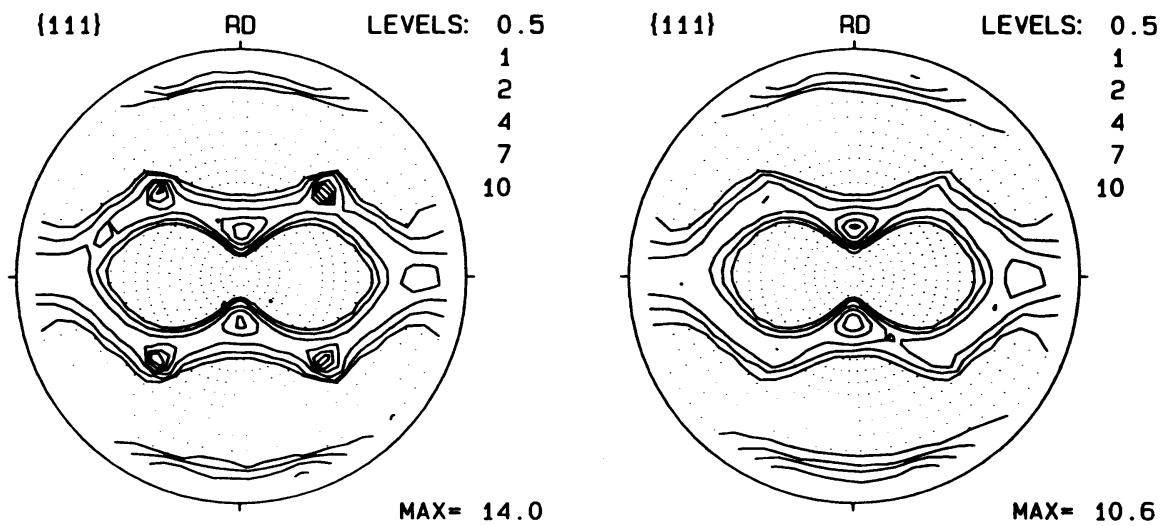

a) $s=0$

b) $s=0,3$
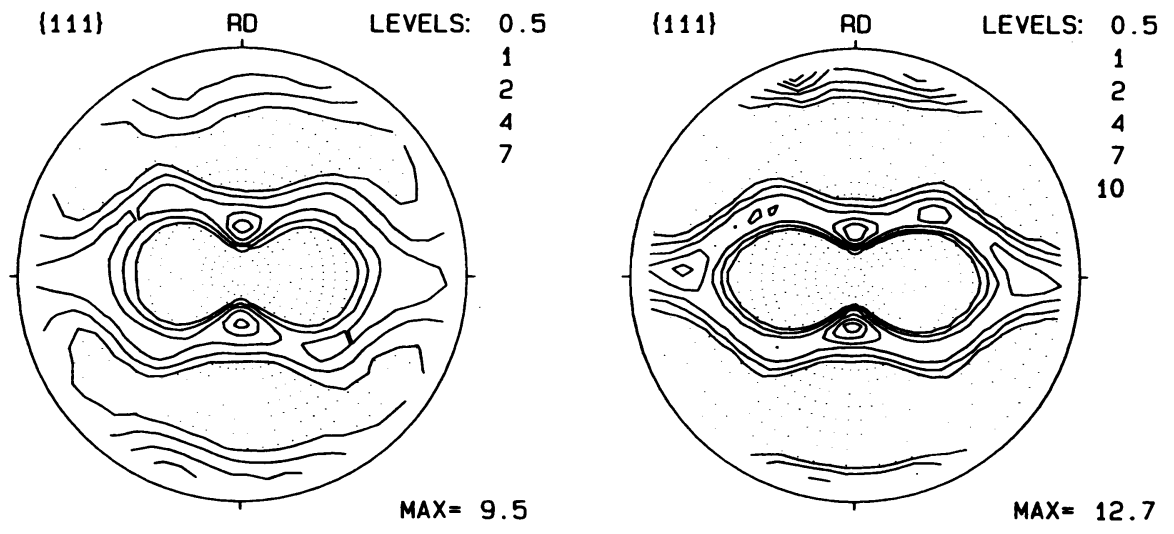

c) $s=0,6$

d) $s=0,9$

Figure $3\{111\}$ pole figures of the rolled sample at various layers determined by X-ray diffraction: a) $s=0, \mathrm{~b}) s=0.3$, c) $s=0.6, \mathrm{~d}) s=0.9$.

absorption of neutrons within the specimen thickness is negligible, neutron experiments directly deliver the averaged texture. These textures look very similar to the averaged X-ray pole figures and ODF's (Figures 5, 6), as already found by Bunge and Tobisch (1968). But they appear more rounded and exhibit somewhat lower peak intensities and larger scattering than the X-ray textures.

\section{(b) Recrystallized Samples}

Figure 8 shows the microstructure of the annealed sheet, revealed again by the $\{111\}$-etch. It possesses a fine grained recrystallized structure with cube (white regions) and cube twin (dark regions) orientations. No differences can be observed for the various depths. 


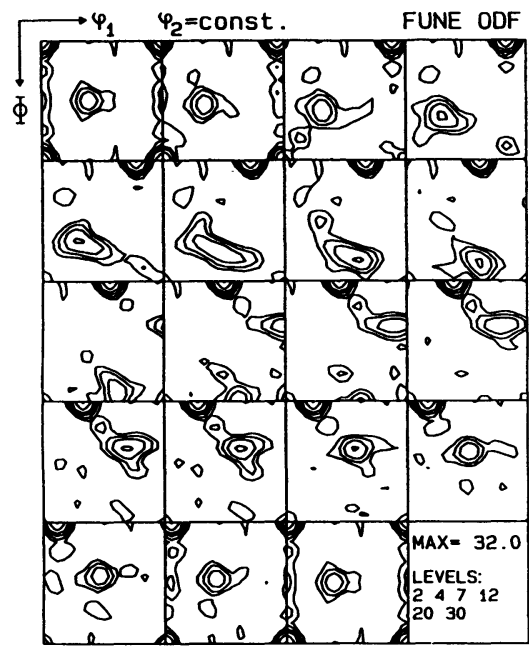

a) $s=0$

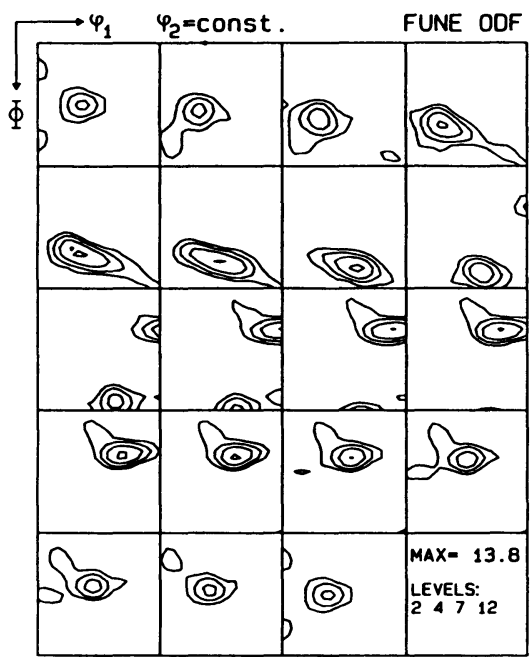

c) $s=0.6$

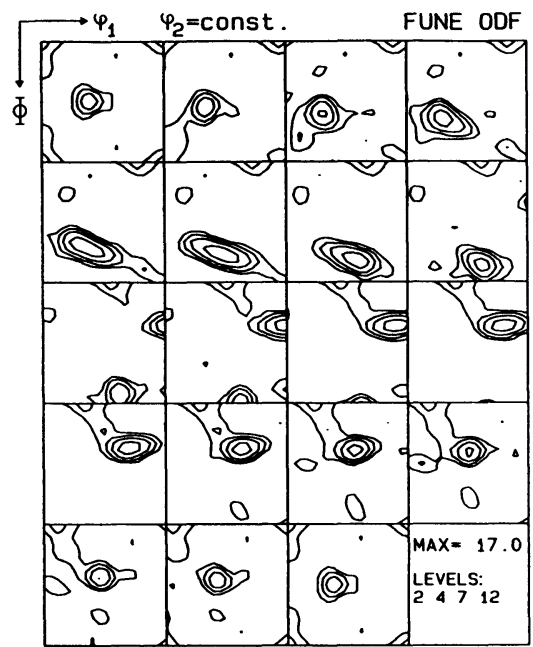

b) $s=0,3$

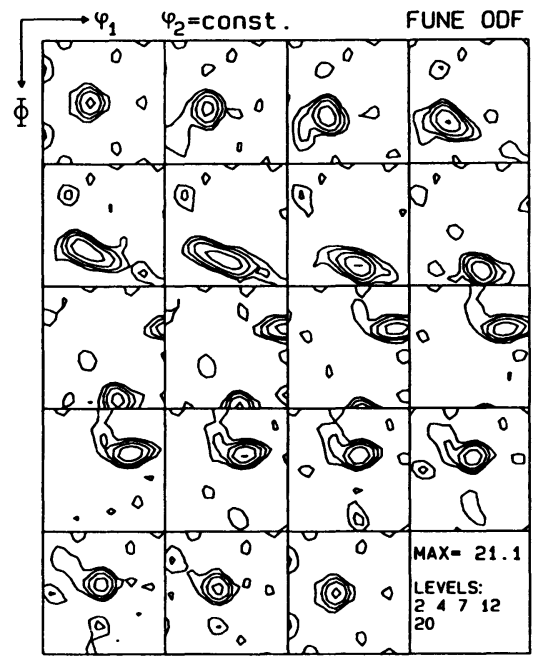

d) $s=0.9$

Figure 4 ODF's of the rolled sample at various layers determined by X-ray diffraction: a) $s=0$, b) $s=0.3$, c) $s=0.6$, d) $s=0.9$.

Figures 9, 10 show the $\{111\}$ pole figures and the ODF's obtained by X-rays from the recrystallized sample for $s=0$ and $s=0.9$ as well as for the sample with the slanted surface. Only very small differences are observed between the pole figures as well as between the corresponding ODF's for the various layers. This indicates a rather homogeneous recrystallization texture. It consists of a strong cube orientation with scattering around the rolling direction and of twins of the 


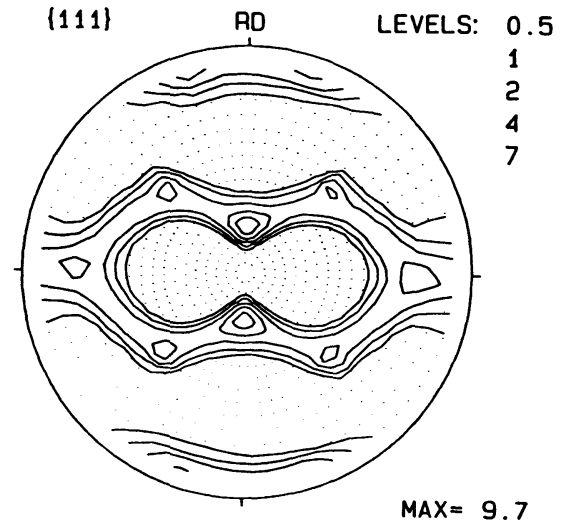

a)

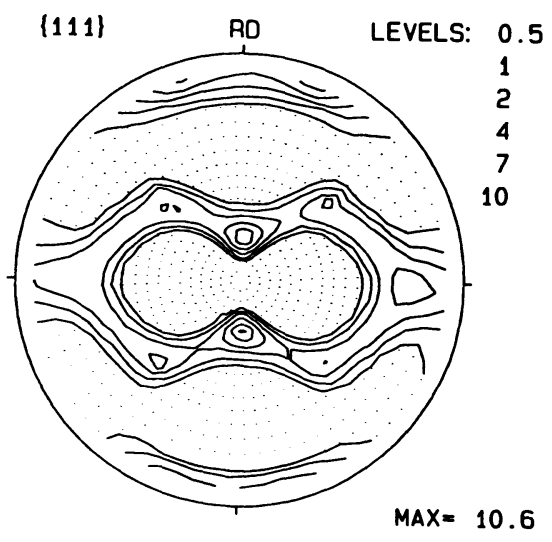

b)

Figure $5\{111\}$ pole figures of the rolled sample determined by X-ray diffraction: a) Numerical averaging of 4 different layers; b) Sample with a slanted surface.

cube. At the mid layer $s=0$ these scatterings are slightly more pronounced than near the sheet surface $s=0.9$.

Figure $11 \mathrm{a}, \mathrm{b}$ show the $\{111\}$ pole figure and ODF obtained by neutron diffraction. They look similar to the corresponding $\mathrm{X}$-ray texture, but, again appear somewhat weaker.

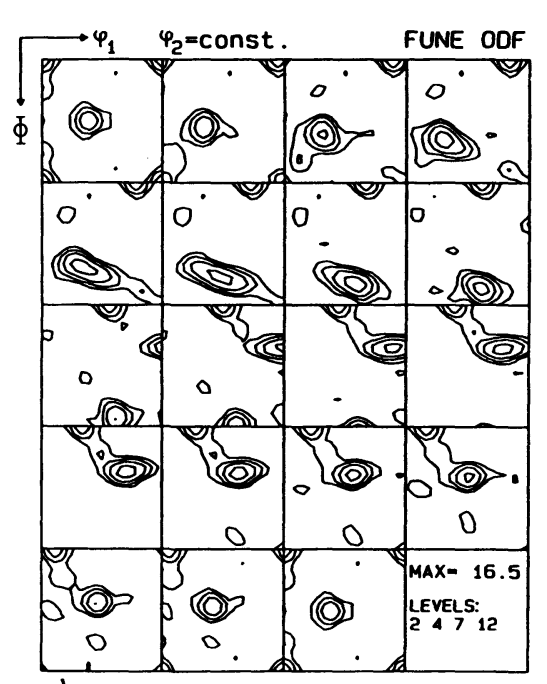

a)

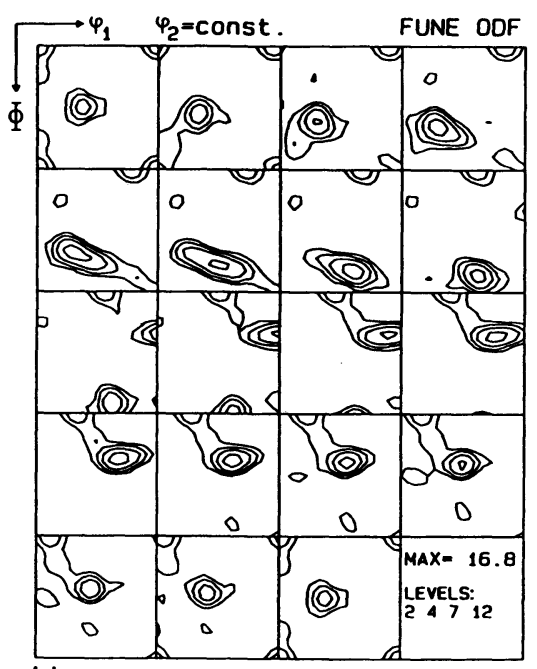

b)

Figure 6 ODF's of the rolled sample determined by X-ray diffraction: a) Numerical averaging of 4 different layers; b) Sample with a slanted surface. 


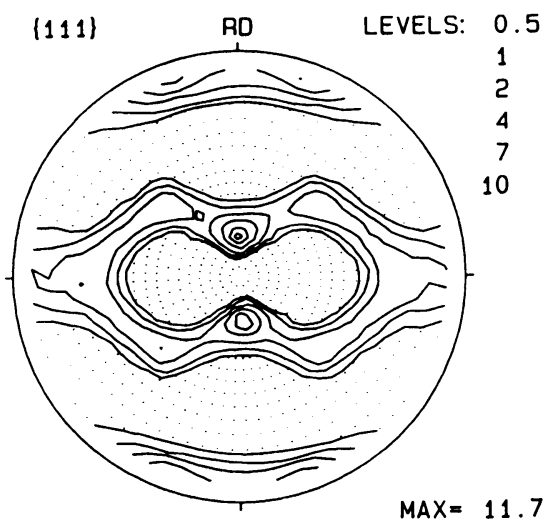

a)

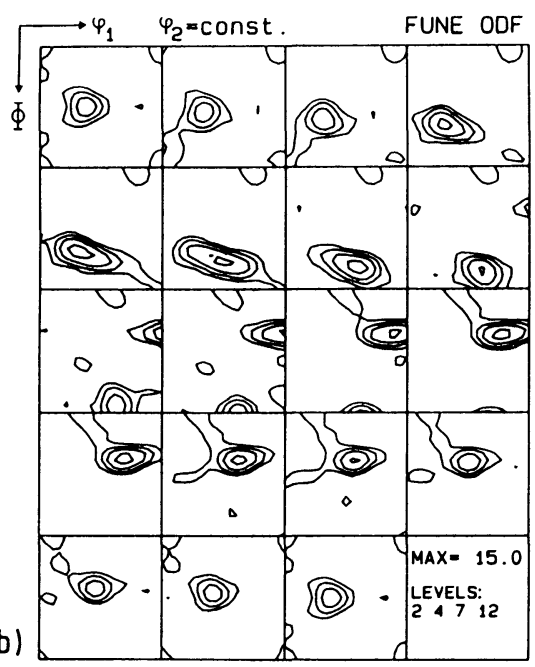

Figure 7 a) $\{111\}$ pole figure and b) ODF of the rolled sample determined by neutron diffraction.

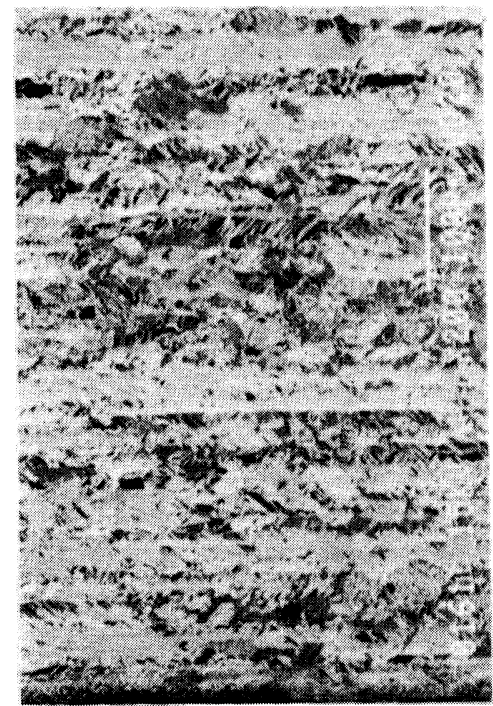

a)

$200 x$
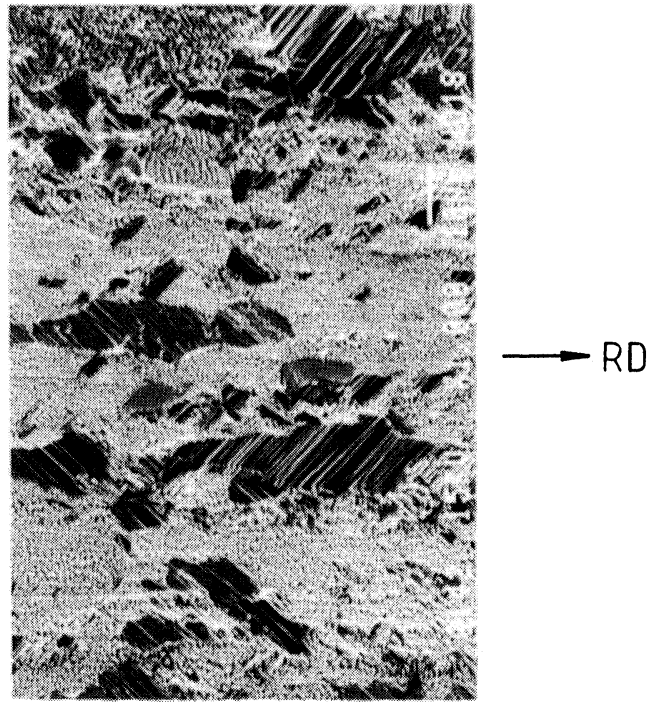

b)

Figure 8 Microstructure of the recrystallized copper sample (longitudinal sections): a) 200×; b) $1000 \times$. 


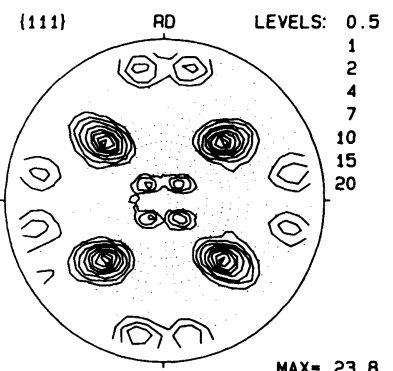

a) $s=0$

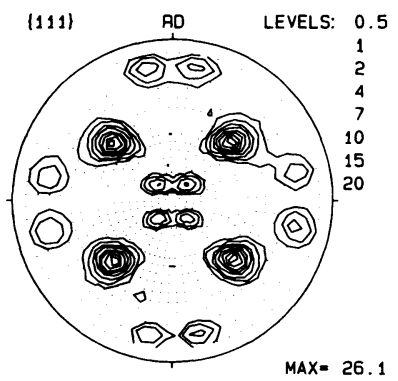

b) $s=0,9$

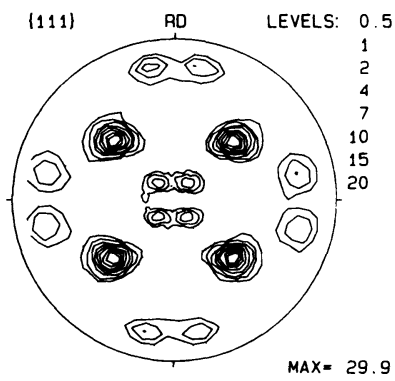

c)

Figure $9\{111\}$ pole figures of the recrystallized sample determined by X-ray diffraction: a) $s=0$; b) $s=0.9 ;$ c) Sample with a slanted surface.
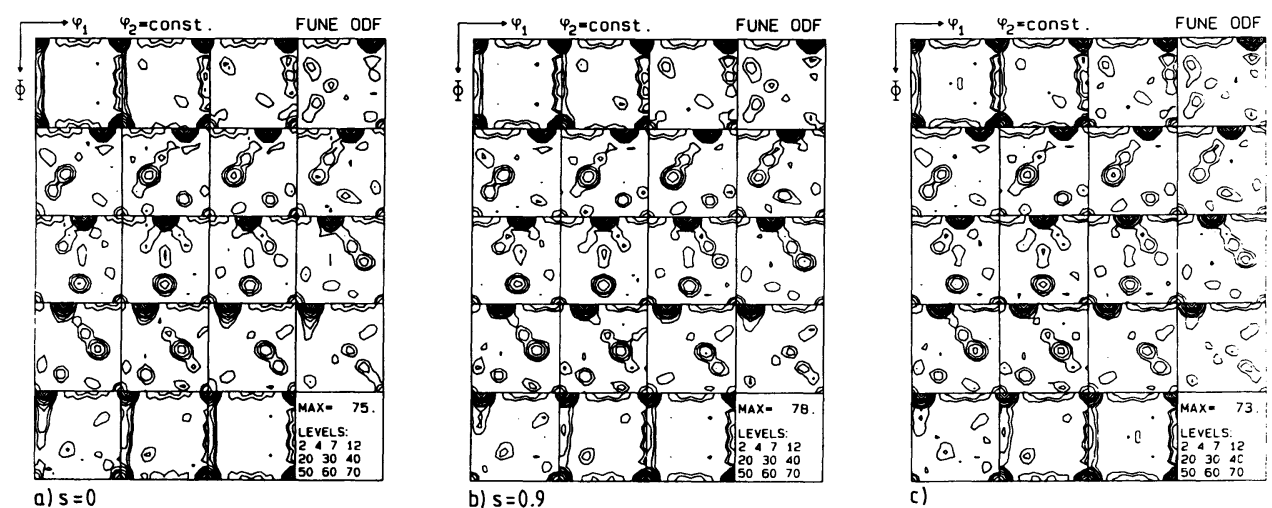

Figure 10 ODF's of the recrystallized sample determined by X-ray diffraction: a) $s=0$; b) $s=0.9$; c) Sample with a slanted surface.

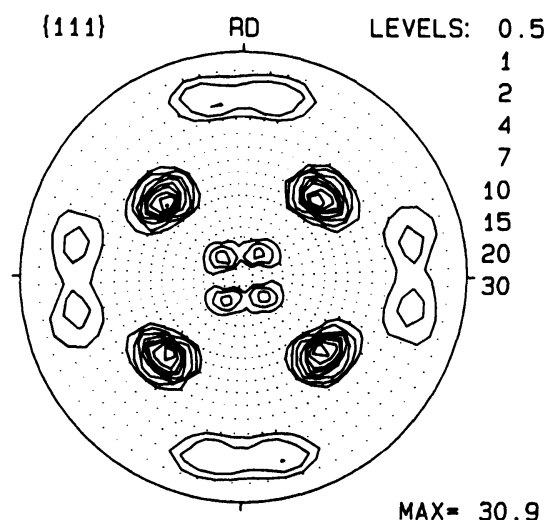

a)

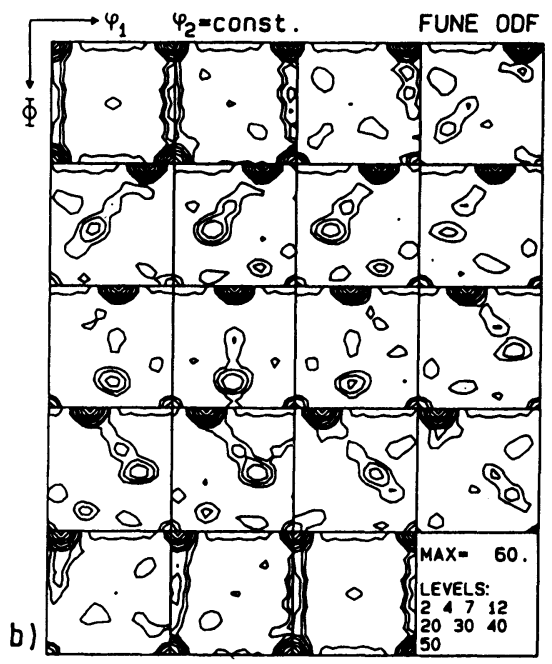

Figure 11 a) $\{111\}$ pole figure and b) ODF of the recrystallized sample determined by neutron diffraction. 


\section{DISCUSSION}

The main differences between the rolling textures obtained from X-ray and neutron measurements are caused by the different penetration depths of the two radiations. In copper, the penetration depth is limited to about $30 \mu \mathrm{m}$ for X-rays compared to several $\mathrm{cm}$ for neutrons. Therefore $\mathrm{X}$-rays have the advantage of possibly yielding more information about textures of various parts of the samplehere of the various layers. Neutron experiments, in contrast, automatically deliver textures integrated over the whole sample, but a separation of different textures occurring in different regions of the sample is principally not possible. In the case of texture variation in the sample, only a superposition of such regional textures is obtained, which may lead to misinterpretations (Pospiech and Lücke, 1978). Finally, because of the larger number of grains met by the neutron beam, they are better suited for statistical evaluations (e.g. for anisotropy calculations), especially in the case of large grain sizes.

Integrated results for samples with texture gradients can also be obtained by $\mathrm{X}$-ray measurements. In the present work two methods were applied. The first method is to measure $\mathrm{X}$-ray textures at various layers and to subsequently average the pole figure data prior to ODF-calculation. But it may be difficult to give the correct weight to the various layers with this method if the textures are rapidly changing. The second method is to prepare a plane at an angle to the sheet surface in a such manner, that all layers $s=0$ to $s=1$ are met by the X-rays (Figure 1). However, preparing such a slanted surface may be rather laborious. Comparing Figures 5, 6 and 7 indeed yields good agreement between the $X$-ray and the neutron results.

In the present study, one set of homogeneous samples, namely the recrystallized ones, and one set of inhomogeneous samples, namely the rolled ones, were investigated. In the latter case, the $\mathrm{X}$-ray measurements revealed cube orientations near the layer $s=0$ embedded by the copper type rolling texture (Figures $3 a, 4 a)$. The existence of the cube component is clearly confirmed by etching (Figure 2) and can safely be assumed to be due to local recrystallization.

The main qualitative result of the present study is that for both, the deformed and the recrystallized samples, the textures obtained by $\mathrm{X}$-rays and by neutrons generally come out to be rather similar, i.e. they are independent of the measuring method. This similarity is more pronounced for the recrystallized (Figures 9-11) than for the rolled samples (Figures 5-7). For the latter ones particularly the cube intensity measured directly on a slanted surface by X-rays comes out to be weaker than that determined by averaging the four layers, but agrees somewhat better with the neutron results. This suggests that in the four layer average the cube texture is overestimated, since it is only present in a thin layer stretching over much less than 1/4 of the sample thickness (Figure 2a).

Quantitatively, the textures derived from the neutron experiments are somewhat less sharp and exhibit more scattering than those stemming from X-ray diffraction (cf. Figures 5-7 and Figures 9, 10). This may be due to the fact that the samples for neutron measurements consist of eleven thin plates being stacked together on top of each other. This stacking introduces small inaccuracies in positioning the exact rolling direction of the various plates which may lead to misorientations of $\sim 1^{\circ}$ causing an increased scattering and, in particular, a slight decrease of the intensity of the texture peaks. Surprisingly, however, the 
maximum intensities of the neutron $\{111\}$ pole figures are higher than those of the X-ray pole figures. Only by considering all four examined pole figures or the ODF's, the maxima measured by means of neutrons are slightly smaller than those stemming from the X-ray experiments.

However, all the intensity differences between pole figures obtained for the same specimen but with different methods lie within a range of $\pm 10 \%$, which must be accepted to be the accuracy of pole figure measurement. Also the somewhat weaker ODF maxima of the neutron textures are still lying within the $\pm 10 \%$ limit. Because of the multitude of influencing parameters an accurate interpretation of these minor intensity differences is difficult. E.g. a slightly modified background correction may result in notable changes of the height of the pole figure maxima. Also the method of pole figure determination, either integrating over the $\Delta \beta=5^{\circ}$ segments of the pole circle (as for the present $X$-ray method) or measuring at discrete values (as for the neutron method) can cause intensity differences. Measuring at discrete points should lead to less pronounced textures, since generally the exact positions of the mimima and maxima are not recorded. Also the interpolation of neutron data might introduce weakening of the obtained ODF's.

\section{CONCLUSIONS}

For the deformed as well as for the recrystallized samples pole figures and ODF's obtained by X-ray and by neutron diffraction are remarkably similar. If texture heterogeneities are present-here for the different layers of the rolled sheet-the results can be compared either by mathematically averaging of the $\mathrm{X}$-ray textures obtained for the different layers or by using samples provided with a new surface at an angle of $\sim 1^{\circ}$ to the original surface. Quantitative comparison proves that the deviations between $X$-ray and neutron textures are less than $10 \%$. This means that the methods used here to correct X-ray data are sufficiently accurate.

\section{ACKNOWLEDGEMENTS}

The authors would like to acknowledge the financial support by the Deutsche Forschungsgemeinschaft (DFG). The neutron diffraction measurements are supported by the Bundesminister für Forschung und Technologie, Bonn, under contract No. 03WI2-BON. One of the authors (J.P.) wants to express his gratitude to the Deutsche Akademische Austauschdienst (DAAD), the Council of Technological Education (COSNET-Mexico City), and the National Council for Science and Technology (CONACYT-Mexico City D111-904642) for funding his collaboration with the German institutes.

\section{References}

Bunge, H. J. (1969). Mathematische Methoden der Texturanalyse, Akademie-Verlag Berlin. Bunge, H. J. (1989). Textures and Microstructures 10, 265-307.

Bunge, H. J., and Tobisch, J. (1968). Z. Metallkde. 59, 471-475. 
Bunge, H. J., Wenk, H. R. and Pannetier, J. (1982). Textures and Microstructures 5, 153-170.

Hammelrath, H., Butler, Jr., J. F., Juul-Jensen, D., Leffers, T., Hu, H., and Lücke, K. (1991). Textures and Microstructures 13, 165-187.

Hirsch, J., Burmeister, G., Hoenen, L., and Lücke, K. (1986). in Experimental Techniques of Texture Analysis, (ed. H. J. Bunge), DGM-Informationsgesellschaft Verlag Oberursel, 63-71.

Hirsch, J., and Lücke, K. (1988). Acta metall. 36, 2863-2927.

Köhlhoff, G., Sun, X., and Lücke, K. (1987). In Textures of Materials, Proc. ICOTOM 8, Sante Fe, (ed. J. S. Kallend, G. Gottstein), the Metallurgical Society, 183-188.

Pospiech, J., and Lücke, K. (1978). Acta metall. 26, 1709-1719.

Schäfer, W., Jansen, E., Kurz, R. and Will, G. (1986). Physica 136B, 110-113.

Schäfer, W., Höfler, S., and Will, G. (1988). Textures and Microstructures 8 \& 9, 457-466.

Tobisch, J. and Bunge, H. J. (1972). Texture 1, 125-127.

Wenk, H. R., Kern, H., Schäfer, W. and Will, G. (1984). Journal of Structural Geology 6, 687-692.

Will, G., Schäfer, W. and Merz, P. (1989). Textures and Microstructures 10, 375-387. 